PREPRINT Accepted for publication.

Cite as: Sandra I. Woolley, Tim Collins; User Experience and Engagement in the Reality-Virtuality Continuum: A Special Issue Guest Editorial. PRESENCE: Virtual and Augmented Reality 2021, pp. 1-4; doi: 10.1162/pres_e_00348

\title{
User Experience and Engagement in the Reality-Virtuality Continuum: A Special Issue Guest Editorial
}

\author{
Sandra I. Woolley ${ }^{1}$ and Tim Collins ${ }^{2}$ \\ ${ }^{1}$ School of Computing and Mathematics, Keele University, UK \\ ${ }^{2}$ Department of Engineering, Manchester Metropolitan University, UK
}

\section{Introduction}

Virtual, augmented and mixed reality technologies are escaping the 'trough of disillusionment' - the lowest point of the Gartner Hype Curve that follows the 'inflated peak of early expectations' (Muñoz-Saavedra et al., 2020). Now, at this exciting juncture, as we see stronger alignments between technology expectations, performance, penetration and application, we are delighted to continue the PRESENCE series of special issues - following on from issues on Arts, Aesthetics, and Performance in Telepresence (Jeon \& Fishwick, 2017), VR for Culture and Heritage: The Experience of Cultural Heritage with Virtual Reality (Ch'ng et al., 2017; Ch'ng et al., 2018), and Virtual and Augmented Reality for Autonomous Driving and Intelligent Vehicles (Riener et al., 2019) - with this new special issue on User Experience and Engagement in the Reality-Virtuality Continuum.

There has, of course, been an international step change in telepresence, virtualization and user experience interest following the Covid-19 pandemic restrictions on travel and inperson leisure, cultural, educational and work-related events and experiences (AmankwahAmoah et al., 2021). Now, we are also seeing virtual technologies and experiences evolve and diversify, for example, ABBA's planned 2022 virtual performances at live venues (BBC, 2021), the creation of dedicated online virtual concert places such as Oberhasli ("Oberhasli," 2021), Apple's anticipated AR/XR technology unveiling (Perry, 2020) and Facebook's 'Meta' rebranding with the launch of its Metaverse as an embodied "evolution in social technology" (Meta, 2021). Perhaps, though, we should be mindful that prior to the pandemic, virtual concerts hosted on popular gaming platforms such as Fortnite and Minecraft, had already attracted millions of attendees (BBC, 2019). All of these exciting developments, together with on-going user experience challenges (Ashtari et al., 2020), inspired this MIT Press PRESENCE journal special issue on User Experience and Engagement in the Reality-Virtuality Continuum.

Our Guest Editor academic interests relating to this special issue theme span two decades of research invested in digitizing and virtualizing cultural artifacts (Ch'ng et al., 2014; Collins et al., 2019) and automating the reconstruction of artifacts from virtual 3D fragments (Woolley et al., 2017). Prior to the outbreak of Covid-19, we were evolving research prototypes of an in-museum QR-code triggered augmented reality 'AR Museum' app for the purpose of supporting and inspiring stakeholder requirement elicitations (Woolley et al., 2020). Like so many research efforts during the pandemic, our project was substantially affected by lockdowns (for us, this was the cancellation of events and the closure of 
UK museums) that meant we needed to redirect our efforts. For example, it was necessary for us to redesign our iOS and Android apps to remove the need for physical triggers and the need for app users to visit specific locations. Perhaps, overall, and in the longer term, the necessitated efforts of all we researchers over these difficult times, will have benefited the evolution of virtualization technologies and applications. Perhaps, also, we researchers will have been further inspired by recent challenges to create more connected and more accessible experiences (Woolley, Collins, Andras, et al., 2021) that could, for example, provide engaging opportunities for 'forgotten' heritage sites (Hain \& Ganobjak, 2018) and for the smaller and less colorful artifacts that are so often overlooked in traditional museum display contexts, where larger and more dramatically visual and colorful objects take center stage (Woolley, Collins, Rhodes, et al., 2021).

\section{About the Issue, Submissions and Review Process}

In this issue we are delighted to bring seven works on User Experience and Engagement in the Reality-Virtuality Continuum to PRESENCE journal readers. These works represent novel and timely contributions from 27 authors at 15 institutions in eight countries. We thank all authors for their valuable contributions to this special issue and we also thank all reviewers who volunteered their time to contribute opinions during what was a very difficult and demanding period.

Launching a journal special issue call for papers during a global pandemic and a series of national lockdowns was not ideal timing, especially given the unprecedented demands on academic time when, for example, there were urgent demands for course virtualization, delivery and assessment, and rather less time for research, paper production and review. Despite these difficulties, papers submitted to this special issue underwent a rigorous review process. Each submitted manuscript was reviewed by two to four reviewers in each of two review rounds. In total, eleven papers were received and seven were accepted. Guest editors performed meta-reviews on the papers in each round of review and the manuscripts selected for publication were those receiving the highest ratings from reviewers.

\section{Papers in this PRESENCE Special Issue}

The seven papers in this special issue on User Experience and Engagement in the Reality-Virtuality Continuum encompass design, analysis and implementation studies addressing virtual reality, augmented reality and hybrid environments.

Two papers relate to interface design relevant to user experience in augmented and virtual realities. For augmented reality interface design, the paper by Joe Cowlyn and Nick Dalton entitled, 'A Spatial Informance Design Method to Elicit Early Interface Prototypes for Augmented Reality' proposes a novel participatory design method for augmented reality interfaces and provides testing evidence demonstrating effectiveness of the method for spa-

tial interface ideation. For virtual environments, the paper by Lisa Rebenitsch and Delaina Engle entitled 'The Effects of Steering Locomotion on User Preference and Accuracy in Virtual Environments', compares virtual environment self-steering methods in terms of accuracy and minimization of cybersickness, evidencing a 'pointing' method using a handheld controller to be preferred in both respects. 
The issue includes two analysis works. 'Lessons Learned from a Study on Distractions in Virtual Learning Environments: Reliability, Ecological Validity and an Elusive Social Component', by Betty Tärning, Eva-Maria Ternblad, Magnus Haake, Agneta Gulz and Jens Nirme, explores the potential for virtual reality to simulate real-life learning situations in educational research and contributes lessons learnt from their study simulating social relationships between the participants and virtual agents and highlighting challenges that need to be addressed to evoke naturalistic behaviors.

Limited expectations and parental resistance toward immersive virtual reality adoption are highlighted and questioned in the empirical work reported in 'When will Immersive Virtual Reality have its Day? Challenges to IVR Adoption in the Home as Exposed in Studies with Teenagers, Parents and Experts', by Lynne Hall, Samiullah Paracha, Nicole Mitsche, Tom Flint, Fiona Stewart, Kate MacFarlane, Gill Hagan-Green and Yvonne DixonTodd. The authors report parental and expert agreement that mainstream immersive virtual reality adoption is unlikely in the near future but, in contrast, expect children to be using headsets in the near future. Teenagers were reported as interested in the technology but did not envisage many applications of interest beyond gaming and entertainment.

In terms of implementation, practice and application, the issue includes three papers. 'Presence and Communication in Hybrid Virtual and Augmented Reality Environments' by Yue Li, Eugene Ch'ng, Sue Cobb and Simon See, explores hybrid environment communication between paired users in virtual and augmented reality environments. Although shared virtual reality experiences were evidenced to afford higher levels of perceived spatial presence, augmented reality users experienced higher levels of perceived social presence with virtual reality partners.

Following on from the 2019 PRESENCE journal special issue on Virtual and Augmented Reality for Autonomous Driving and Intelligent Vehicles (Riener et al., 2019), this special issue further contributes to the domain with 'A Systematic Review of Augmented Reality Applications for Automated Driving: 2009 - 2020' from Andreas Riegler, Andreas Riener and Clemens Holzmann. In this review, 156 papers are surveyed and categorized, and trends identified, for example, the trend toward simulating augmented reality content in virtual driving simulators.

Remote learning became a necessity during the Covid lockdowns; Cecilia Hammar Wijkmark, Ilona Heldal and Maria Metallinou provide a technology assessment in 'What Makes Remote Virtual Simulation for Practice-Based Training Work? - Presence and Performance in Incident Commander Education'. They provide detailed experimental observations, including some positive experiences and outcomes, in the transition from live on-site simulation to remote virtual simulation training for Swedish Fire Service Incident Commanders.

\section{Concluding Remarks}

We greatly appreciate the contributions of all 27 special issue authors and of all reviewers. We cordially invite PRESENCE readers to a fascinating journey through a collection of high-quality research articles assembled in this special issue on User Experience and Engagement in the Reality-Virtuality Continuum.

Guest Editors: Sandra I. Woolley, School of Computing and Mathematics, Keele University, UK, and Tim Collins, Department of Engineering, Manchester Metropolitan 
University, UK

\section{References}

Amankwah-Amoah, J., Khan, Z., Wood, G., \& Knight, G. (2021). Covid-19 and digitalization: The great acceleration. Journal of Business Research, 136, 602-611.

Ashtari, N., Bunt, A., McGrenere, J., Nebeling, M., \& Chilana, P. K. (2020). Creating augmented and virtual reality applications: Current practices, challenges, and opportunities. Proceedings of the 2020 CHI Conference on Human Factors in Computing Systems, 1-13.

BBC. (2019). Fortnite: 'millions attend' virtual Marshmello concert [Last Accessed Nov 1st, 2021.]. https://www.bbc.co.uk/news/blogs-trending-47116429

BBC. (2021). Abba delight fans with new 10-song album and virtual concert [Last Accessed Nov 1st, 2021.]. https://www.bbc.co.uk/news/entertainment-arts-58423452

Ch'ng, E., Cai, Y., \& Thwaites, H. (2017). Special issue on VR for culture and heritage: The experience of cultural heritage with virtual reality: Guest editors' introduction. Presence: Teleoperators and Virtual Environments, iii-vi.

Ch'ng, E., Cai, Y., \& Thwaites, H. (2018). Special issue on VR for culture and heritage: The experience of cultural heritage with virtual reality (part II): Guest editors' introduction. Presence: Teleoperators and Virtual Environments, iii-iv.

Ch'ng, E., Woolley, S. I., Hernandez-Munoz, L., Collins, T., Lewis, A., \& Gehlken, E. (2014). The development of a collaborative virtual environment for 3D reconstruction of cuneiform tablets. 2014 International Conference on Virtual Systems 83 Multimedia (VSMM), 35-42. https://doi.org/10.1109/VSMM.2014.7136692

Collins, T., Woolley, S. I., Gehlken, E., \& Ch'ng, E. (2019). Automated low-cost photogrammetric acquisition of 3D models from small form-factor artefacts. Electronics, 8(12), 1441. https://doi.org/10.3390/electronics8121441

Hain, V., \& Ganobjak, M. (2018). Forgotten industrial heritage in virtual reality-case study: Old power plant in Piešt'any, Slovakia. Presence, 26(4), 355-365. https: //doi.org/10.1162/pres_a_00309

Jeon, M. “, \& Fishwick, P. (2017). Special issue on arts, aesthetics, and performance in telepresence: Guest editors' introduction: Homo ludens in virtual environments. Presence: Teleoperators and Virtual Environments, 26(2), iii-vii.

Meta. (2021). Introducing Meta: A social technology company, press release [Last Accessed Nov 1st, 2021.]. https://about.fb.com/news/2021/10/facebook-company-is-nowmeta/

Muñoz-Saavedra, L., Miró-Amarante, L., \& Domínguez-Morales, M. (2020). Augmented and virtual reality evolution and future tendency. Applied sciences, 10(1), 322. https: //doi.org/10.3390/app10010322

Oberhasli [Last Accessed Nov 1st, 2021.]. (2021). https://www.coregames.com/games/ $09 \mathrm{e} 277 /$ oberhasli

Perry, T. S. (2020). Look out for Apple's AR glasses: With head-up displays, cameras, inertial sensors, and lidar on board, Apple's augmented-reality glasses could redefine wearables. IEEE Spectrum, 58(1), 26-54. 
Riener, A., Gabbard, J., \& Trivedi, M. (2019). Special issue of presence: Virtual and augmented reality virtual and augmented reality for autonomous driving and intelligent vehicles: Guest editors' introduction. Presence, 27, i-iv. https://doi.org/10.1162/ pres_e_00323

Woolley, S. I., Collins, T., Andras, P., Gardner, A., Ortolani, M., \& Pitt, J. (2021). Compounding barriers to fairness in the digital technology ecosystem. IEEE International Symposium on Technology and Society (ISTAS), Oct 2021, Waterloo, Ontario, Canada.

Woolley, S. I., Collins, T., Rhodes, R., \& Polack, F. (2021). Museums of the future: Heritage experiences in the reality-virtuality continuum. BCS Proceedings of the British HCI Conference, July 2021 London, UK.

Woolley, S. I., Ch'ng, E., Munoz, L. U. H., Gehlken, E., Collins, T., Nash, D., Lewis, A., \& Hanes, L. (2017). A collaborative artefact reconstruction environment. Proceedings of the 31st International BCS Human Computer Interaction Conference, BCS HCI 2017, Sunderland, UK, 3-6 July 2017. https://doi.org/10.14236/ewic/HCI2017.53

Woolley, S. I., Mitchell, J., Collins, T., Rhodes, R., Rukasha, T., Gehlken, E., Ch'ng, E., \& Cooke, A. (2020). Virtual museum 'takeouts' and DIY exhibitions-augmented reality apps for scholarship, citizen science and public engagement. Euro-Mediterranean Conference, 323-333. https://doi.org/10.1007/978-3-030-73043-7_27 